\title{
Performance of Optimum Combining in a Poisson Field of Interferers and Rayleigh Fading Channels
}

\author{
Olfa Ben Sik Ali, Christian Cardinal, Member, IEEE, \\ and François Gagnon, Senior Member, IEEE
}

\begin{abstract}
This paper studies the performance of antenna array processing in distributed multiple access networks without power control. The interference is represented as a Poisson point process. Desired and interfering signals are subject to both path-loss fading (with an exponent greater than 2) and to independent Rayleigh fading. Using these assumptions, we derive the exact closed form expression for the cumulative distribution function of the output signal-to-interference-plus-noise ratio when optimum combining is applied. This results in a pertinent measure of the network performance in terms of the outage probability, which in turn provides insights into the network capacity gain that could be achieved with antenna array processing. We present and discuss examples of applications, as well as some numerical results.
\end{abstract}

\section{Index Terms}

Multiple access network, Poisson point process, shot noise process, power decay law, Rayleigh fading, MMSE linear combining, optimum combining, outage probability, and capacity.

O. Ben Sik Ali and C. Cardinal are with the Department of Electrical Engineering, École Polytechnique, Montréal, CA, (e-mail: \{olfa.ben-sik-ali, christian.cardinal\}@ polymtl.ca).

F. Gagnon is with the Department of Electrical Engineering, École de Technologie Supérieure, Montréal, CA, (e-mail: Francois.Gagnon@etsmtl.ca ). 


\section{INTRODUCTION}

In wireless communications, energy and spectrum resources are scarce and expensive, and must consequently be managed efficiently in order to address the growing requirements of emerging applications. Intensive work has been dedicated to developing advanced processing technologies to improve the spectral efficiency. In decentralized multiple access systems, such as ad hoc networks, interference is the major performance inhibitor, which explains why in recent years, much research effort has been dedicated to the interference mitigation. Several solutions have been proposed involving exploitation of the particular structure of the interference. In fact, technologies such as spread spectrum and multiple antennas can be used to design systems with a number of degrees of freedom that, when properly exploited, increase system tolerance to interference. In the context of spread spectrum, various multiuser receiver schemes have been introduced [1]. The well-known minimum mean square error (MMSE) receiver is the linear multiuser receiver that maximizes the signal-to-interference-plus-noise ratio (SINR), and the equivalent solution in the context of antenna array processing is known as the optimum combining (OC) receiver [2]. Though the study of the reliability of these receivers is an important issue, which has been the subject of extensive work [3]-[6], there is still a lot of work left to do in order to understand their limits in various contexts. In fact, the bulk of the results available deal with special assumptions concerning interference, such as considering equal power interferers, strong interferers or asymptotic cases (infinite number of interferers and antennas). In this paper, we address the issue of quantifying the performance gain that could be achieved by employing the optimum combining receiver in a decentralized network without using power or access control.

\section{A. Problem statement and contribution}

As previously mentioned, we are interested in distributed random access networks. A common representation of this kind of networks is to consider a random number of interferers that are independently and identically distributed over the area. The number of interferers is measured by one parameter, namely, their spatial density. Thus, we adopt the so-called stochastic geometric model which is widely used in the literature for the access layer as well as for the physical layer [7]-[9]. In this model, the link outage is defined as the probability that the expected SINR seen by a representative receiver is below a certain threshold. The expectation is taken over the set of possible realizations of the network. As defined, the outage probability serves to derive several 
spatial performance metrics, namely, the mean number of transmissions in outage per unit area, the mean throughput per unit area, and the mean distance covered by all transmissions per unit area. From these spatial performance metrics, we can study the connectivity, the transport capacity and several important issues of the network performance. The tutorial paper [10] provides a survey of these issues and discusses some applications of this model and this methodology to wireless communication.

To derive the outage probability, we need the interference distribution. In a stochastic geometric network, the interference is a spatial shot-noise process with an impulse response having a decaying power law form. Even with one antenna and the matched filter receiver, the distribution of the interference does not take a closed form (except for some special cases, such as a path loss exponent equal to 4) although its characteristic function is well known [11]-[14].

Recent works have focused on analyzing the performance of more advanced receivers than the conventional one. In [8], [15] and [16], approximations and bounds on the outage probability are given for the successive interference cancellation receiver. In the context of smart antennas, [17] gives lower and upper bounds on the outage probability for sectorized antenna, maximal ratio combining and space time coding techniques. Analyses of the zero-forcing and the partial zero-forcing receivers follow in [18], [19]. The main contribution of the work presented in this paper is the exact derivation of the outage probability for the optimum combining receiver with an arbitrary number of antennas.

\section{B. Organization of the paper}

The outline for this paper is as follows. In Section II, we present the system model. The derivation of the outage probability, which is the main result obtained in this paper, is presented in Section III. Applications and simulations follow in Sections IV and V, and section VI concludes the paper.

\section{SySTEM MODEL}

\section{A. Network and channel model}

In a distributed network, a receiver is surrounded by a number of undesired source nodes that transmit on the same medium and in the same time slot as its intended transmitter. Usually, receiver nodes do not control the number and the positions of these sources of interference; 
rather, these are determined by the network dynamics and the access strategy in place. In the particular case of a random access strategy, a simple way to model the network is to consider interfering nodes distributed according to a homogeneous Poisson point process (PPP) [7], [20]. A PPP relies on a single parameter $\lambda$, namely, the density of transmitters. In a planar network, it is described as follows: In a closed region with area $A$, the number of transmitters is distributed according to the Poisson law with density $\lambda A$. The positions of these nodes are uniformly distributed on the plane. We assume that each node transmits with a single antenna and receives with $L$ antennas. The distance between an emitting node and its intended receiver is set to $d_{r}$. Formally, the set of emitting nodes forms a homogeneous PPP $\Pi=\left\{X_{i} \in \mathbb{R}^{2}, \lambda, i \in \mathbb{N}\right\}$, where $X_{i}$ are the nodes' locations. The channel is modeled by two independent components, the first of which represents the path-loss attenuation with an exponent $\alpha>2$, and the second, represents the channel coefficients, which are independent among antennas and nodes.

\section{B. Interference expression under antenna array processing}

Since the network is modeled as a homogenous PPP, the interference distribution does not depend on the spatial locations of receivers. Thus, in the following we focus, without any loss of generality, on a representative receiver placed at the origin. A receiver is subject to a number of interfering signals coming from non-desired transmitters. The received signal vector is then:

$$
\mathbf{x}=d_{r}^{-\alpha / 2} \mathbf{c}_{r} s_{r}+\sum_{X_{k} \in \Pi}\left|X_{k}\right|^{-\alpha / 2} \mathbf{c}_{k} s_{k}+\mathbf{n},
$$

where $\mathbf{c}_{r}$ and $\mathbf{c}_{k}, k \in \mathbb{N}$, are the propagation vectors with dimension $L$ that have independently and identically distributed, zero-mean, unit variance complex Gaussian entries. The vector $\mathbf{n}$ is a zero-mean complex Gaussian noise with variance $\sigma^{2}$ per dimension. All nodes are supposed to use the same transmission power, normalized to unity.

In statistical antenna array processing, a weight vector is chosen based on the statistics of the data received and optimized under a given criterion. Hence, the output of the antenna array processor is:

$$
\mathbf{y}=\mathbf{w}^{T} \mathbf{x}
$$


where $\mathbf{w}$ is a complex weight vector. The operator ${ }^{T}$ denotes the transpose conjugate. Several criteria could be considered in determining this vector [2], such as a maximization of the desired signal-to-noise ratio corresponding to the maximal ratio combining receiver. In systems with interference, the aim is to optimize the antenna array output such that the quantity of noise and interference is minimized in the resulting signal. In other words, maximizing the SINR:

$$
\mathbf{w}_{O C}=\arg \max _{\mathbf{w}}\left\{\frac{\mathbf{w}^{T} \mathbf{c}_{r} \mathbf{c}_{r}^{T} \mathbf{w}}{\mathbf{w}^{T}\left(\mathbf{R}_{I}+\sigma^{2} \mathbf{I}_{L}\right) \mathbf{w}}\right\},
$$

where $\mathbf{I}_{L}$ is the $L \times L$ identity matrix and $\mathbf{R}_{I}$ is the interference covariance matrix expressed as $\mathbf{R}_{I}=\sum_{X_{k} \in \Pi}\left|X_{k}\right|^{-\alpha} \mathbf{c}_{k} \mathbf{c}_{k}^{T}$. The well-known solution corresponds to [2], [21]:

$$
\mathbf{w}_{O C}=\left(\mathbf{R}_{I}+\sigma^{2} \mathbf{I}_{L}\right)^{-1} \mathbf{c}_{r}
$$

The resulting SINR is denoted as $\beta$ and expressed as:

$$
\beta=d_{r}^{-\alpha} \mathbf{c}_{r}^{T} \mathbf{R}^{-1} \mathbf{c}_{r}
$$

where $\mathbf{R}$ is the interference plus noise covariance matrix expressed as $\mathbf{R}=\mathbf{R}_{I}+\sigma^{2} \mathbf{I}_{L}$.

\section{OUTAGE PROBABILITY DERIVATION}

The configuration we consider has two random parameters, namely, the locations of interferers and the channel coefficients. Thus, we have a complex probabilistic system, which we propose to break down into two levels. First, we generate the SINR expression conditioned on a realization of the network, after which we average over this random quantity. Consider an arbitrary planar region $D$ with finite radius $d$ and arbitrary nodes positions, and use $N$ to denote the number of nodes in place. The problem now is to find the distribution (or the eigenvalue distribution) of random quadratic matrices having the form $\left(\mathbf{C P C}^{T}+\sigma^{2} \mathbf{I}_{L}\right)$. Each column of $\mathbf{C}$ represents a channel vector of an interferer. Thus, the random matrix $\mathbf{C}$ has column vectors independently and identically distributed as multivariate normals with zero-mean and unit-variance vectors. The matrix $\mathbf{P}=\operatorname{diag}\left[\left|X_{1}\right|^{-\alpha},\left|X_{2}\right|^{-\alpha}, \cdots\right]$ is a diagonal matrix with real elements corresponding to the set of received powers. 


\section{A. Related results}

The problem stated in the previous paragraph is a classic problem in the probability and wireless communication literature. We can classify the most pertinent results to the context of this work into two categories. The first category concerns the work of Silverstein and Bai [22], who consider an asymptotic regime (the number of nodes and the number of antennas tend to infinity at a fixed rate). They established that the eigenvalues of the considered class of random matrices converge to a deterministic limiting distribution. This result is first used in [23] and [24] to derive a closed form expression of the asymptotic mean and variance of the SINR at the output of the MMSE receiver, in the case of deterministic received powers. In [25], the authors apply these asymptotic techniques to the particular case of emitting nodes uniformly distributed on an infinite plane, and provide the SINR mean and variance. Following this methodology, [26] gives an approximation of the SINR distribution based on the assumption that the latter is a Gamma distribution. In our case, despite the large number of users (the network area is wide), these results cannot be applied. In fact, the spatial separations of users ensures that the global interference is only influenced by a small number of them [8], [15], [16]. In addition, the goal of our work is to show how using a small number of receive antennas can improve the SINR. The second category was initiated by Khatri [27], who derived the distribution of the matrix $\mathrm{CPC}^{T}$. From this, [28] provides the SIR distribution when the noise is ignored:

$$
f_{\beta}(\beta)=\frac{\Gamma(N+1)}{\Gamma(L) \Gamma(N+1-L)} \frac{\beta^{L-1} q^{N+1}}{(1+q \beta)^{N+1}}|\mathbf{P}|^{-1} \mathrm{H}_{0}^{(N)}\left(N+1 ; \mathbf{I}_{N}-q \mathbf{P}^{-1}, \mathbf{Z}\right),
$$

where $H_{0}^{(N)}$ is a hypergeometric function of matrix arguments, $\mathbf{Z}=\operatorname{diag}\left[(1+q \beta)^{-1}, \mathbf{I}_{L-1}\right], q$ is a particular constant and $\Gamma(\cdot)$ is the gamma function.

Even if we consider that our system is interference-limited, expression (6) is not simple as it contains the hypergeometric function with matrix arguments. It therefore appears to be difficult to derive the SINR distribution when $\mathbf{P}$ is a random matrix. In [29] and [30], the authors extended the previous result by including the noise term and derived a simpler expression. The derivation consists in expressing the hypergeometric function as a polynomial ratio. The cumulative distribution function (CDF) of the SINR is given as:

$$
F_{\beta}\left(\beta \mid N, X_{1} \cdots X_{N}\right)=1-\frac{\sum_{i=0}^{L-1} a_{i}\left(\beta d_{r}^{\alpha}\right)^{i}}{\exp \left(\sigma^{2} \beta d_{r}^{\alpha}\right) \prod_{j=1}^{N}\left(1+\left|X_{j}\right|^{-\alpha} \beta d_{r}^{\alpha}\right)},
$$


where $a_{i}, i=0 \cdots L-1$, are the first $L$ coefficients of the Taylor expansion of $\exp \left(\sigma^{2} \beta\right) \prod_{j=1}^{N}(1+$ $\left.\left|X_{j}\right|^{-\alpha} \beta\right)$.

\section{B. Outage probability expression}

First, let us consider the case of a single receive antenna that corresponds to a Rayleigh fading channel. In this case, as established by [9], the complementary CDF of the SINR is the characteristic function of the sum of interference and noise, which is a perfect match for expression (7). In fact, $\prod_{j=1}^{N}\left(1+\left|X_{j}\right|^{-\alpha} \gamma\right)^{-1}$ is the characteristic function expression of a weighted sum (with weights equal to $\left|X_{j}\right|^{-\alpha}$ ) of $N$ independently and exponentially distributed random variables. Since the characteristic function of a Poisson class of interferers is well known [11]-[14], [20], [31], [32], the derivation for $L=1$ is trivial. For an arbitrary number of antennas, taking the expectation over the possible realizations of the network and denoting $\beta d_{r}^{\alpha}$ by $\gamma$, we establish the following theorem.

Theorem 1: Using the MMSE receiver, the outage probability in a Poisson field of interferers and Rayleigh fading channel is:

$$
F(\gamma, \lambda)=1-\sum_{i=0}^{L-1} \frac{\left(\lambda \Delta \gamma^{2 / \alpha}+\sigma^{2} \gamma\right)^{i}}{i !} \exp \left(-\lambda \Delta \gamma^{2 / \alpha}-\sigma^{2} \gamma\right)
$$

where $\Delta=\pi 2 / \alpha \Gamma(2 / \alpha) \Gamma(1-2 / \alpha)$.

Proof: The coefficients $a_{i}$ in (7) can be derived simply. In fact, extending the exponential in the denominator of (7) and putting $P_{j}=\left|X_{j}\right|^{-\alpha}$ we get:

$$
\exp \left(\sigma^{2} \gamma\right) \prod_{j=1}^{N}\left(1+P_{j} \gamma\right)=\sum_{k=0}^{\infty} \frac{\left(\sigma^{2}\right)^{k}}{k !} \gamma^{k} \sum_{i=0}^{N} b_{i}\left(P_{1}, \cdots, P_{N}\right) \gamma^{i}
$$

where $b_{i}\left(P_{1}, \cdots, P_{N}\right)=\sum_{1 \leq j_{1}<\cdots<j_{i} \leq N} P_{j_{1}} P_{j_{2}} \cdots P_{j_{i}}$. Then, $a_{i}$ are expressed as follows:

$$
a_{i}=\sum_{k=0}^{i} \frac{\left(\sigma^{2}\right)^{i-k}}{(i-k) !} b_{k}\left(P_{1}, \cdots, P_{N}\right) \quad i=1 \cdots L-1
$$

From (7) and (10):

$$
F_{\gamma}(\gamma, \lambda)=1-\exp \left(-\sigma^{2} \gamma\right) \mathrm{E}_{N}\left[\sum_{i=0}^{L-1} \sum_{k=0}^{\min (i, N)} \frac{\left(\sigma^{2}\right)^{i-k}}{(i-k) !} \gamma^{i-k} \mathrm{E}_{P_{1}, \cdots, P_{N}}\left[\frac{b_{k}\left(P_{1}, \cdots, P_{N}\right) \gamma^{k}}{\prod_{j=1}^{N}\left(1+P_{j} \gamma\right)}\right]\right],
$$


where $\mathrm{E}_{x}$ denotes the expectation with respect to the random variable $x$. The coefficients $b_{k}\left(P_{1}, \cdots, P_{N}\right), k=1 \cdots L-1$, are composed of a sum of products of combinations of $k$ elements from the set $\left\{P_{j}, j=1 \cdots N\right\}$. Given that the locations of the nodes are independently and identically distributed, the last expression simplifies to yield:

$$
\begin{aligned}
\mathrm{E}_{P_{1}, \cdots, P_{N}}\left[\frac{b_{k}\left(P_{1}, \cdots, P_{N}\right) \gamma^{k}}{\prod_{j=1}^{N}\left(1+P_{j} \gamma\right)}\right] & =C_{N}^{k} \mathrm{E}_{P_{1}, \cdots, P_{N}}\left[\frac{P_{1} \cdots P_{k} \gamma^{k}}{\prod_{j=1}^{k}\left(1+P_{j} \gamma\right)} \frac{1}{\prod_{j=k+1}^{N}\left(1+P_{j} \gamma\right)}\right] \\
& =C_{N}^{k} \mathrm{E}_{P_{1}}\left[\frac{P_{1} \gamma}{\left(1+P_{1} \gamma\right)}\right]^{k} \mathrm{E}_{P_{1}}\left[\frac{1}{\left(1+P_{1} \gamma\right)}\right]^{N-k},
\end{aligned}
$$

where $C_{N}^{k}$ is the number of combinations of size $k$ from a set of $N$ elements. To compute $\mathrm{E}_{P_{1}}\left[\frac{P_{1}}{\left(1+P_{1} \gamma\right)}\right]$, recall that conditioned on $\mathrm{N}$, in a finite region $D \in \mathbb{R}^{2}$, the locations of nodes are uniformly distributed. Consequently:

$$
\mathrm{E}_{P_{1}}\left[\frac{P_{1}}{\left(1+P_{1} \gamma\right)}\right]=\frac{1}{\pi d^{2}} \int_{D}|X|^{-\alpha}\left(1+|X|^{-\alpha} \gamma\right)^{-1} \mathrm{~d} X .
$$

Similarly, we have:

$$
\mathrm{E}_{P_{1}}\left[\frac{1}{\left(1+P_{1} \gamma\right)}\right]=\frac{1}{\pi d^{2}} \int_{D}\left(1+|X|^{-\alpha} \gamma\right)^{-1} \mathrm{~d} X
$$

Since the number of nodes is distributed according to the Poisson law with mean $\lambda \pi d^{2}$, and considering (13) and (14), the CDF of the SINR yields:

$$
\begin{aligned}
F_{\gamma}(\gamma, \lambda)= & 1-\exp \left(-\sigma^{2} \gamma\right) \sum_{N=0}^{\infty} \sum_{i=0}^{L-1} \sum_{k=0}^{\min (i, N)} \frac{N !}{k !(N-k) !(i-k) !}\left(\sigma^{2}\right)^{i-k} \gamma^{i-k}\left(\frac{1}{\pi d^{2}} \int_{D} \frac{|X|^{-\alpha} \gamma}{1+|X|^{-\alpha} \gamma} \mathrm{d} X\right)^{k} . \\
& \left(\frac{1}{\pi d^{2}} \int_{D} \frac{\mathrm{d} X}{1+|X|^{-\alpha} \gamma}\right)^{N-k} \frac{\left(\lambda \pi d^{2}\right)^{N}}{N !} \exp \left(-\lambda \pi d^{2}\right) \\
= & 1-\exp \left(-\sigma^{2} \gamma\right) \sum_{i=0}^{L-1} \sum_{k=0}^{i} \sum_{N=k}^{\infty} \frac{\left(\sigma^{2} \gamma\right)^{i-k}}{k !(i-k) !}\left(\lambda \int_{D} \frac{|X|^{-\alpha} \gamma}{1+|X|^{-\alpha} \gamma} \mathrm{d} x\right)^{k} . \\
& \frac{1}{(N-k) !}\left(\lambda \int_{D} \frac{\mathrm{d} X}{1+|X|^{-\alpha} \gamma}\right)^{N-k} \exp \left(-\lambda \pi d^{2}\right) .
\end{aligned}
$$

Using the fact that $\pi d^{2}=\int_{D} 1 \mathrm{~d} X$, we get:

$F_{\gamma}(\gamma, \lambda)=1-\exp \left(-\sigma^{2} \gamma\right) \sum_{i=0}^{L-1} \sum_{k=0}^{i} \frac{1}{k !(i-k) !}\left(\sigma^{2} \gamma\right)^{i-k}\left(\lambda \int_{D} \frac{|X|^{-\alpha} \gamma}{1+|X|^{-\alpha} \gamma} \mathrm{d} X\right)^{k} \exp \left(\lambda \int_{D}\left(\frac{1}{1+|X|^{-\alpha} \gamma}-1\right) \mathrm{d} X\right)$. 
Finally, letting the area of $D$ go to infinity and evaluating the integrals in (16) (the complete evaluation of these integrals is provided in the Appendix) we get:

$$
\begin{aligned}
F_{\gamma}(\gamma, \lambda) & =1-\exp \left(-\sigma^{2} \gamma\right) \sum_{i=0}^{L-1} \sum_{k=0}^{i} \frac{1}{k !(i-k) !}\left(\sigma^{2} \gamma\right)^{i-k}\left(\gamma^{2 / \alpha} \lambda \Delta\right)^{k} \exp \left(-\gamma^{2 / \alpha} \lambda \Delta\right) \\
& =1-\sum_{i=0}^{L-1} \frac{\left(\lambda \Delta \gamma^{2 / \alpha}+\sigma^{2} \gamma\right)^{i}}{i !} \exp \left(-\lambda \Delta \gamma^{2 / \alpha}-\sigma^{2} \gamma\right) .
\end{aligned}
$$

\section{DisCUSSION AND APPLICATION}

\section{A. Noise-limited regime and interference-limited regime}

The expression (8) derived for the outage probability has a simple form. From it, we can easily see the effects of various parameters on the network performance. Moreover, it clearly presents the trade-off between noise and interference cancellation, and thus provides some insight into the intuition behind the result.

1) Noise-limited regime: In systems where the density of users is negligible, the SNR cumulative distribution function becomes:

$$
F_{\gamma}(\gamma)=1-\sum_{i=0}^{L-1} \frac{\left(\sigma^{2} \gamma\right)^{i}}{i !} \exp \left(-\sigma^{2} \gamma\right)
$$

As expected, (18) is the classic expression of a Chi-square cumulative distribution function. The latter provides the SNR distribution when maximal ratio combining is performed, that is equivalent to optimum combining in a complex Gaussian noise environment.

2) Interference-limited regime: Where the noise is negligible, the $\mathrm{CDF}$ is:

$$
F_{\gamma}(\gamma, \lambda)=1-\sum_{i=0}^{L-1} \frac{\left(\lambda \Delta \gamma^{2 / \alpha}\right)^{i}}{i !} \exp \left(-\lambda \Delta \gamma^{2 / \alpha}\right)
$$

Equation (19) corresponds, up to the factor $\Delta$, to the probability that the $L^{\text {th }}$ largest received power is below the threshold $\gamma$ [33]. This is not surprising given some properties of the interference. Since the path loss exponent is greater than 2, the interference distribution is heavy tailed [34]. Therefore, there is a large dispersion between the received powers. Let us order the received powers and denote them as $\left|X_{(1)}\right|^{-\alpha} \gg\left|X_{(2)}\right|^{-\alpha} \gg\left|X_{(3)}\right|^{-\alpha} \gg \cdots$. With probability one, the 
random matrix $\mathrm{CPC}^{T}$ is of order $L$, and its eigenvalues could be approximated as $\left|X_{(i)}\right|^{-\alpha}$, $i=1 \cdots L[35]$. Thus the maximal eigenvalue of the inverse of $\mathbf{C P C}^{T}$ is approximately $\left|X_{(L)}\right|^{\alpha}$. The performance is then primarily affected by the $L^{\text {th }}$ strongest received power, and so, the outage is almost due to the $L^{t h}$ strongest interferer, and corresponds to the event $\left|X_{(L)}\right|<\gamma^{1 / \alpha}$. In other words, to achieve successful reception, the $L^{\text {th }}$ strongest interferer must be outside the region of radius $\gamma^{1 / \alpha}$. This fact matches expression (19) up to the scalar factor $\Delta$.

3) SIR mean and variance: From (19), we can establish in a straightforward manner that in an interference-limited regime, the mean and the variance of the SIR are, respectively:

$$
\begin{gathered}
E[S I R]=\frac{\Gamma(L+\alpha / 2)}{(L-1) !} \frac{d_{r}^{-\alpha}}{\lambda^{\alpha / 2} \Delta^{\alpha / 2}}, \\
\operatorname{Var}[S I R]=\left(\frac{\Gamma(L+\alpha)}{(L-1) !}-\left[\frac{\Gamma(L+\alpha / 2)}{(L-1) !}\right]^{2}\right) \frac{d_{r}^{-2 \alpha}}{\lambda^{\alpha} \Delta^{\alpha}} .
\end{gathered}
$$

Thus, the MMSE receiver provides an antenna array gain equal to $\Gamma(L+\alpha / 2) /(L-1)$ !. It should be noted that expression (19) is consistent with the result previously established in [25]. In fact, when the number of antennas is sufficiently high, the mean SIR is approximately equal to $L^{\alpha / 2} \frac{d_{r}^{-\alpha}}{\lambda^{\alpha / 2} \Delta^{\alpha / 2}}$. This latter relation is also derived for the asymptotic regime, i.e., very large $L$, in [25].

\section{B. Application: single-hop throughput capacity}

The interference model considered serves to capture several network classes under some additional assumptions. In the following, we focus on a single-hop ad hoc network with an ALOHA access protocol. Thus, the density of interferers represents the spatial rate at which transmissions occur, i.e., the contention density. In this context, the outage probability represents the spatial average of the density of communications that fail to be established at a given range $d_{r}$ in almost every given realization of the network [9]. Equally, the mean number of successful transmissions or the throughput per unit area is $T=\lambda(1-F(\gamma, \lambda))$. The simplicity of our analytical result allows the direct optimization of the contention density as:

$$
\lambda_{\max }=\arg \max _{0 \leq \lambda<\infty} \lambda(1-F(\gamma, \lambda))
$$

Corollary 1: In an interference-limited regime, the optimum contention density is:

$$
\lambda_{\max }=\frac{g(L)}{\Delta \gamma^{2 / \alpha}} .
$$


The parameter $g(L)$ is only a function of the number of receive antennas and corresponds to the positive root of the following polynomial:

$$
Q(t)=\sum_{i=0}^{L-1} \frac{t^{i}}{i !}-\frac{t^{L}}{(L-1) !}
$$

Moreover, this parameter satisfies:

$$
\frac{L}{2} \leq g(L) \leq L
$$

The equivalent spatial throughput per unit area is:

$$
T_{\text {max }}=\frac{g^{L+1}(L)}{(L-1) ! \Delta \gamma^{2 / \alpha}} \exp (-g(L)) .
$$

Corollary 1 indicates that the MMSE receiver provides a linear scaling of the optimum contention density with the number of receive antennas unlike to the MRC and the zero-forcing receivers, whose scaling laws are $L^{2 / \alpha}$ and $L^{1-2 / \alpha}$, respectively [17], [18]. The linear scaling law achieved by the MMSE receiver is predicted in [19]. In fact, the authors show that linear scaling is possible with a partial zero-forcing receiver, which is suboptimal as compared to the MMSE receiver. Through corollary 1, we confirm this prediction, and we show that the scaling law is exactly linear.

\section{Simulation Results}

All simulations are carried out with the following parameters: path-loss coefficient $\alpha=3.5$, SINR threshold $\beta=3 d B$ and distance between transmitter and receiver $d_{r}=10 \mathrm{~m}$. Figure 1 shows the simulation and the analytical results of the outage probability as a function of the density $\lambda$ where 1,2,3 and 4 antennas are used. The Monte Carlo simulation and the analytical curves are very close, with the gap between them arising from the fact that the analysis performed on the previous section concerns infinite networks. In our simulations, the area is finite, and depends on the density $\lambda$. It is chosen such that we have 100 emitters in the area, on average. In [36], the author provides a complete analysis of the error on the interference estimation when a finite network is considered rather than an infinite one, and establishes that this error depends on the exponent $\alpha$.

Figure 2 provides a performance comparison between the optimal combining receiver and three antenna array processing techniques, namely, maximal ratio combining, zero-forcing and partial zero-forcing receivers. It is clear that the optimum combining receiver outperforms the other 
techniques. This is due to the fact that maximal ratio combining deals only with the fading effects of the desired signal. Zero-forcing receiver uses all additional degrees of freedom provided by the antennas to cancel strong interferers, while the partial zero-forcing receiver uses some of the antennas for interference cancellation and provides diversity with the remaining ones; they are nevertheless still suboptimal as compared to the MMSE receiver, which provides the best trade-off between interference cancellation and spatial diversity.

Figure 3 presents the throughput density improvement with the number of antennas and figure 4 shows the linear scaling of the optimum contention density.

\section{CONCLUSION}

This paper derived the exact outage probability of the optimum combining receiver in the presence of noise and Poisson field of interferers. The framework developed is nonspecific and can be generalized in a straightforward manner to any fading having a power distribution of the form $\sum_{k} x^{k} e^{-k x}$, such as the Nakagami fading. The result obtained has a simple closed form, and provides an understanding of the performance of the MMSE receiver, in addition to allowing a comparison with other antenna array processing methods, such as maximal ratio combining and zero-forcing receivers. The assumption made on the interference is pertinent to many network classes. In addition, the result can be used to study the performance of the optimum combining receiver at the access and at the network layers. Finally, the simulations provided in the paper demonstrate that experimental and theoretical results match perfectly.

\section{APPENDIX A}

EVALUATION OF THE INTEGRALS IN (16)

The integrals in (16) are evaluated as follows:

$$
\begin{aligned}
\int_{\mathbb{R}^{2}}\left(\left(1+|X|^{-\alpha} \gamma\right)^{-1}-1\right) \mathrm{d} X & =\int_{0}^{\infty} \int_{0}^{2 \pi}\left(\left(1+r^{-\alpha} \gamma\right)^{-1}-1\right) r \mathrm{~d} \theta \mathrm{d} r \\
& =-2 \pi \int_{0}^{\infty} \frac{r^{-\alpha+1}}{1+r^{-\alpha} \gamma} \mathrm{d} r
\end{aligned}
$$

Putting $y=r^{\alpha} \gamma$, the last expression becomes: 


$$
\begin{aligned}
\int_{\mathbb{R}^{2}}\left(\left(1+|X|^{-\alpha} \gamma\right)^{-1}-1\right) \mathrm{d} X & =-2 \pi \frac{\gamma^{2 / \alpha}}{\alpha} \int_{0}^{\infty} \frac{y^{1-2 / \alpha}}{1+y} \mathrm{~d} y \\
& =-2 \pi \frac{\gamma^{2 / \alpha}}{\alpha} \Gamma(2 / \alpha) \Gamma(1-2 / \alpha) \\
& =-\gamma^{2 / \alpha} \Delta .
\end{aligned}
$$

On the other hand, we have

$$
\int_{\mathbb{R}^{2}} \frac{|X|^{-\alpha} \gamma}{1+|X|^{-\alpha} \gamma} \mathrm{d} x=-\int_{\mathbb{R}^{2}}\left(\left(1+|X|^{-\alpha} \gamma\right)^{-1}-1\right) \mathrm{d} x
$$

\section{APPENDIX B}

\section{PROOF OF COROLLARY 1}

By Descartes' rule of signs, the polynomial in expression (24) has at most one real positive root. The value of this polynomial at $L$ is negatively signed:

$$
\begin{aligned}
Q(L) & =\sum_{i=0}^{L-1} \frac{L^{i}}{i !}-\frac{L^{L}}{(L-1) !} \\
& =\sum_{i=0}^{L-1}\left[\frac{L^{i}}{i !}-\frac{L^{L}}{L !}\right] \\
& =\sum_{i=0}^{L-1}\left[\frac{L^{i}}{i !}\left(1-\frac{L^{L-i}}{(i+1) \cdots L}\right)\right] \\
& \leq 0
\end{aligned}
$$

On the other hand, the value of the polynomial at $L / 2$ is lower bounded as follows:

$$
\begin{aligned}
Q(L / 2) & =\sum_{i=0}^{L-1} \frac{L^{i}}{2^{i} i !}-\frac{L^{L}}{2^{L}(L-1) !} \\
& \geq \sum_{i=0}^{L-1} C_{L}^{i} \frac{1}{2^{i}}-\frac{L^{L}}{2^{L}(L-1) !} \\
& \geq\left(\frac{3}{2}\right)^{L}-\frac{1}{2^{L}}-\frac{L^{L}}{2^{L}(L-1) !}
\end{aligned}
$$

The right hand side of inequality (31) is positive for all values of $L$. Thus, the considered polynomial has one positive root that lies in the interval $[L / 2, L]$. 


\section{ACKNOWLEDGMENT}

\section{The authors would like to thank Dr. Sergey Loyka for his helpful comments and suggestions.}

\section{REFERENCES}

[1] S. Verd, Multiuser Detection. Cambridge: Cambridge University Press, 1998.

[2] C. A. Baird and C. Zham, "Performance criteria for narrowband array processing," in IEEE Conference On Decision And Control, vol. 10, 1971, pp. 564-565.

[3] J. H. Winters, "Optimum combining in digital mobile radio with cochannel interference," IEEE J. Select. Areas Commun., vol. 2, no. 4, pp. 528-539, 1984.

[4] V. A. Aalo and J. Zhang, "Performance of antenna array systems with optimum combining in a rayleigh fading environment," IEEE Commun. Lett., vol. 24, no. 12, pp. 387-389, 2000.

[5] D. Lao and A. Haimovich, "Exact closed-form performance analysis of optimum combining with multiple cochannel interferers and rayleigh fading," IEEE Trans. Commun., vol. 51, no. 6, pp. 995-1003, 2003.

[6] M. Chiani, M. Win, and A. Zanella, "On optimum combining of M-aray PSK signals with unequal power interferers and noise," IEEE Trans. Commun., vol. 53, no. 1, pp. 44-47, 2005.

[7] E. Sousa, "Performance of a spread spectrum packet radio network link in a Poisson field of interferers," IEEE Trans. Inform. Theory, vol. 38, no. 6, pp. 1743-1754, 1992.

[8] S. Weber, J. Andrews, X. Yang, and G. D. Veciana, "Transmission capacity of wireless ad hoc networks with successive interference cancellation,” IEEE Trans. Inform. Theory, vol. 53, no. 8, pp. 2799-2814, 2007.

[9] F. Baccelli, B. Blaszczyszyn, and P. Muhlethaler, "An aloha protocol for multihop mobile wireless networks," IEEE Trans. Inform. Theory, vol. 52, no. 2, pp. 421-436, 2006.

[10] M. Haenggi, J. G. Andrews, F. Baccelli, O. Dousse, and M. Franceschetti, "Stochastic geometry and random graphs for the analysis and design of wireless networks," IEEE J. Select. Areas Commun., vol. 27, no. 7, pp. 1029-1046, 2009.

[11] S. O. Rice, "Mathematical analysis of random noise," Bell System Technical Journal, vol. 23, pp. 1-51, 1944.

[12] W. B. Davenport and W. L. Root, An Introduction to the Theory of Random Signals and Noise. John Wiley \& Sons, 1987.

[13] S. B. Lowen and M. C. Teich, "Power law shot noise,” IEEE Trans. Inform. Theory, vol. 23, no. 6, pp. 1302-1318, 1990.

[14] D. L. Synder and M. I. Miller, Random Point Processes in Time and Space, 2nd ed. Spring Verlag.

[15] O. Ben-Sik-Ali, B. L. Agba, C. Cardinal, and F. Gagnon, "Performances simulation of flat fading channels in mobile ad hoc networks," in International Symposium on Signals, Systems and Electronics ISSSE'07, pp. 189-191.

[16] V. Mordachev and S. Loyka, “On node density outage probability tradeoff in wireless networks," in IEEE ISIT'08, 2008.

[17] A. M. Hunter, J. Andrews, and S. Weber, "Transmission capacity of ad hoc networks with spatial diversity," IEEE Trans. Wireless Commun., vol. 7, no. 12, pp. 5058-5071, 2008.

[18] K. Huang, J. Andrews, R. Heath, D. G, and R. Berry, "Spatial interference cancellation for multi-antenna mobile ad hoc networks: Perfect CSI," in IEEE GLOBECOM'08, 2008.

[19] N. Jindal, S. P. Weber, and J. Andrews, "Rethinking MIMO for wireless networks: Linear throughput increases with multiple receiver antenna," in IEEE ICC'09, 2009.

[20] J. Ilow and D. Hatzinakos, "Analytic alpha stable noise modeling in a poisson field of interferers or scatterers," IEEE Trans. Signal Processing, vol. 46, no. 6, pp. 1601-1611, 1998. 
[21] H. Cox, R. M. Zeskind, and M. M. Owen, “Robust adaptive beamforming,” IEEE Trans. Acoust., Speech, Signal Processing, vol. 35, pp. 1365-1375, 1987.

[22] J. W. Silverstein and Z. D. Bai, "On the empirical distribution of eigenvalues of a class of large dimensional random matrices," Journal of Multivariate Analysis, vol. 54, no. 2, pp. 786-795, 1994.

[23] D. Tse and S. Hanly, "Linear multiuser receiver: Effective interference, effective bandwidth and user capacity," IEEE Trans. Inform. Theory, vol. 45, no. 2, pp. 641-657, 1999.

[24] S. verdu and S. Shamai, "Multiuser detection with random spreading and error correction codes: Fundamental limits," in Allerton'97, 1997.

[25] S. Govindasamy, D. W. Bliss, and D. H. Staelin, "Spectral efficiency in single-hop ad hoc wireless networks with interference using adaptive antenna arrays,” IEEE J. Select. Areas Commun., vol. 25, no. 7, pp. 1358-1369, 2007.

[26] J. Ma, Y. J. Zhang, X. Su, and Y. Yao, “On capacity of wireless ad hoc networks with MIMO MMSE receivers," vol. 7, no. 12 , pp. 5493-5503, 2008.

[27] C. G. Khatri, "On certain distribution problems based on positive definite quadratic functions in normal vectors," Annals of Mathematical Statistics, vol. 37, no. 2, pp. 468-479, 1966.

[28] A. Shah and M. Haimovich, "Performance analysis of optimum combining in wireless communications with rayleigh fading and cochannel interference," IEEE Trans. Commun., vol. 46, no. 4, pp. 473-479, 1998.

[29] H. Gao and P. J. Smith, "Exact SINR calculations for optimum linear combining in wireless systems," Probability in the engineering and Informational Sciences, vol. 40, no. 1, pp. 261-281, 1998.

[30] H. Gao, P. J. Smith, and M. V. Clark, "Theoretical reliability of MMSE linear diversity combining in rayleigh fading additive interference channels," IEEE Trans. Commun., vol. 46, no. 5, pp. 666-672, 1998.

[31] W. Schottky, "Uber spontane stromschwankungen in verschiedenen elektrizitatsleitern," Annalen der physik, vol. 57, pp. 541-567, 1918.

[32] A. P. Petropulu and J. C. Pesquet, "Power law shot noise and its relationship to long memory $\alpha$-stable processes," IEEE Trans. Signal Processing, vol. 48, no. 7, pp. 1883-1892, 2000.

[33] H. A. . David and H. N. Nagaraja, Order Statistics, 3rd ed. John Wiley \& Sons, 2003.

[34] R. Adler, R. Feldman, and M. Taqqu, A Practical Guide to Heavy Tails: Statistical Techniques and Applications. Boston: Birkhauser, 1998.

[35] E. Villier, "Performance analysis of optimum combining with multiple interferers in flat rayleigh fading," IEEE Trans. Commun., vol. 47, no. 10, pp. 1503-1510, 1999.

[36] J. Ilow, "Signal processing in alpha-stable noise environments: Noise modeling, detection and estimation," Ph.D. dissertation, Univ. of Toronto, Canada, 1996. 


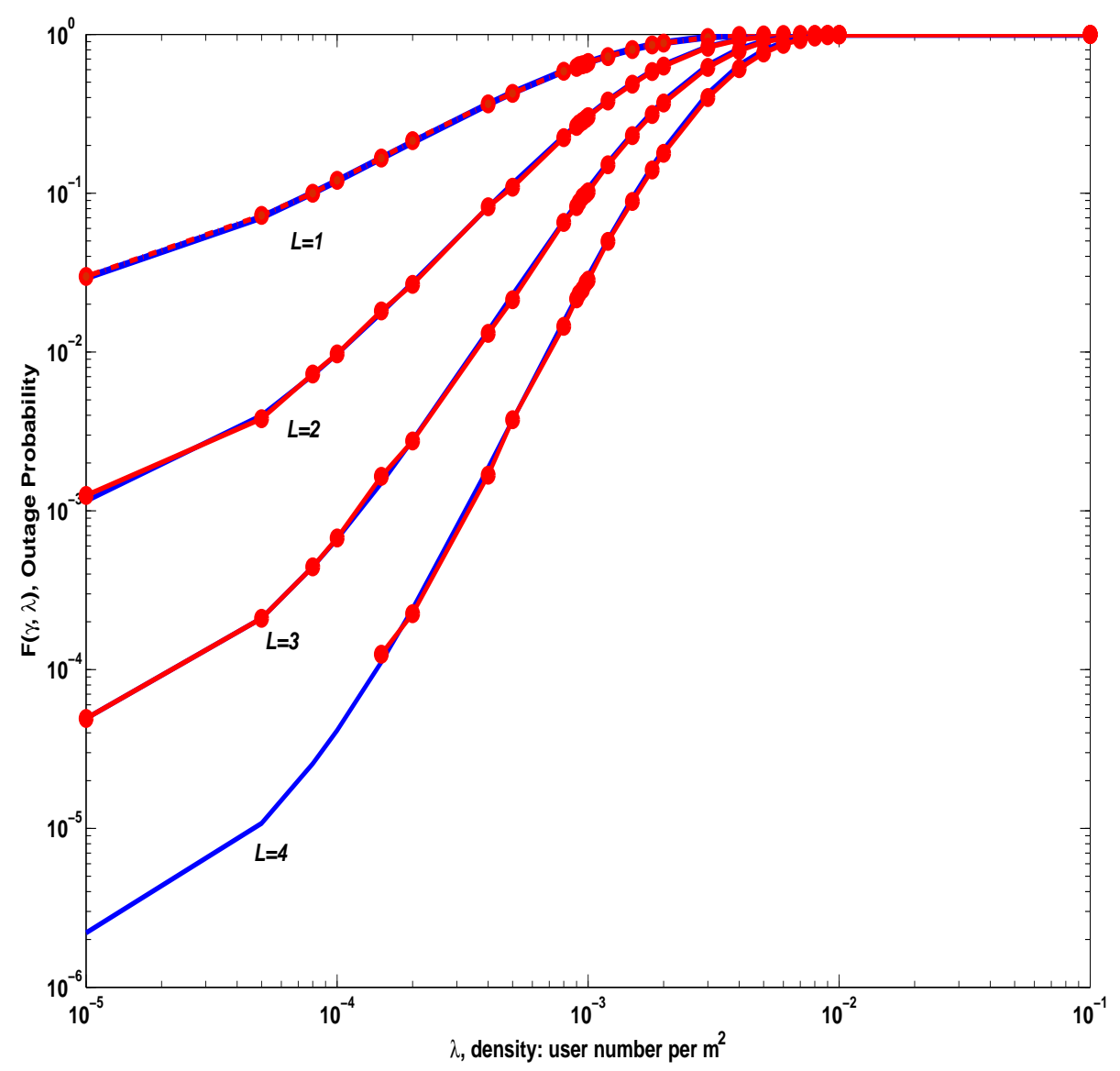

Fig. 1: Outage probability: simulation (dashed lines) and theoretical (solid lines) results with $\sigma^{2}=-50 d B /$ antenna (all nodes use unit transmission power.) 


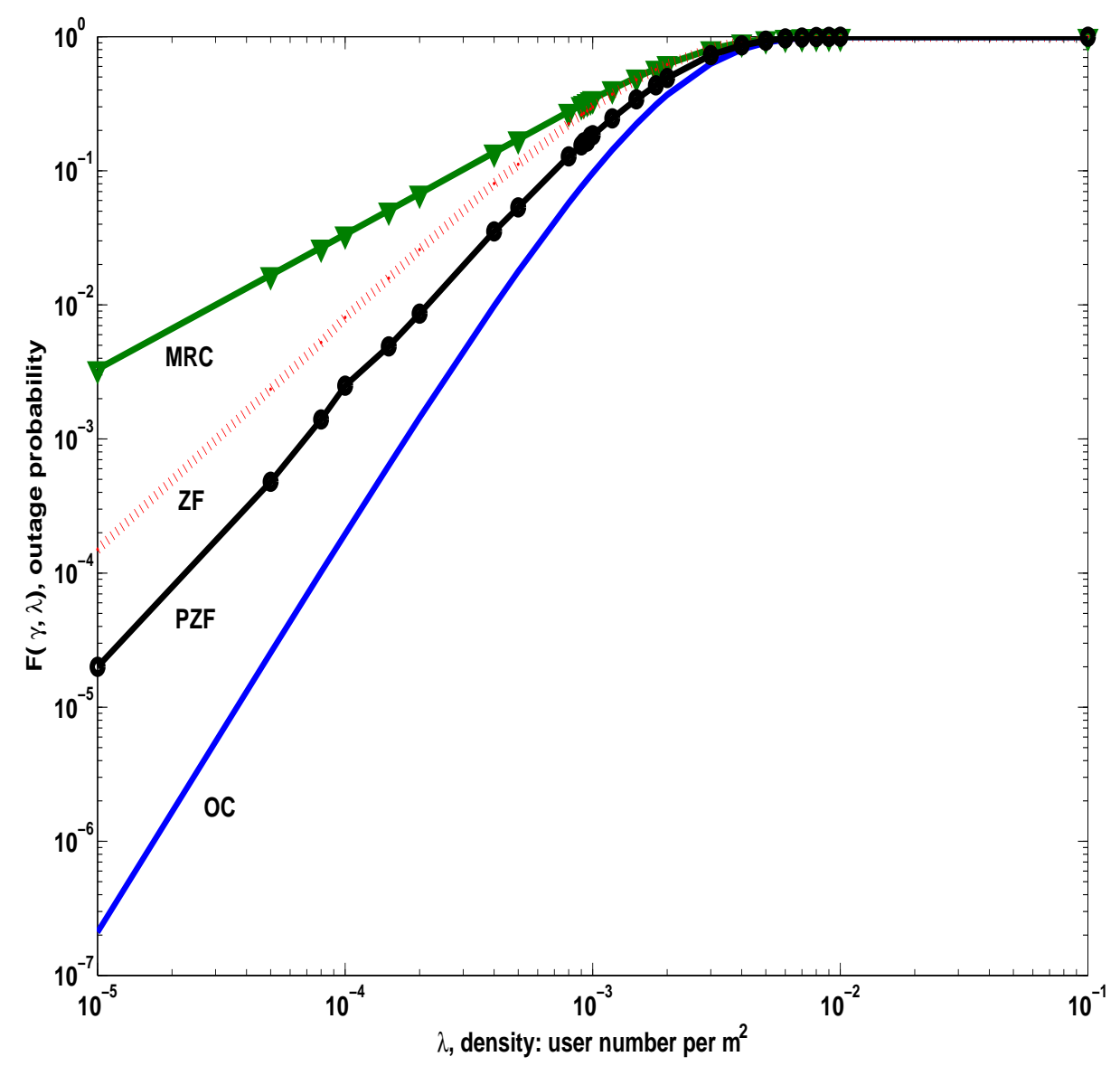

Fig. 2: Outage probability: Maximal ratio combining, zero forcing, partial zero forcing (with the optimum number of cancelled interferers established in [19]) and optimum combining with $L=3$ and $\sigma^{2}=0$. 


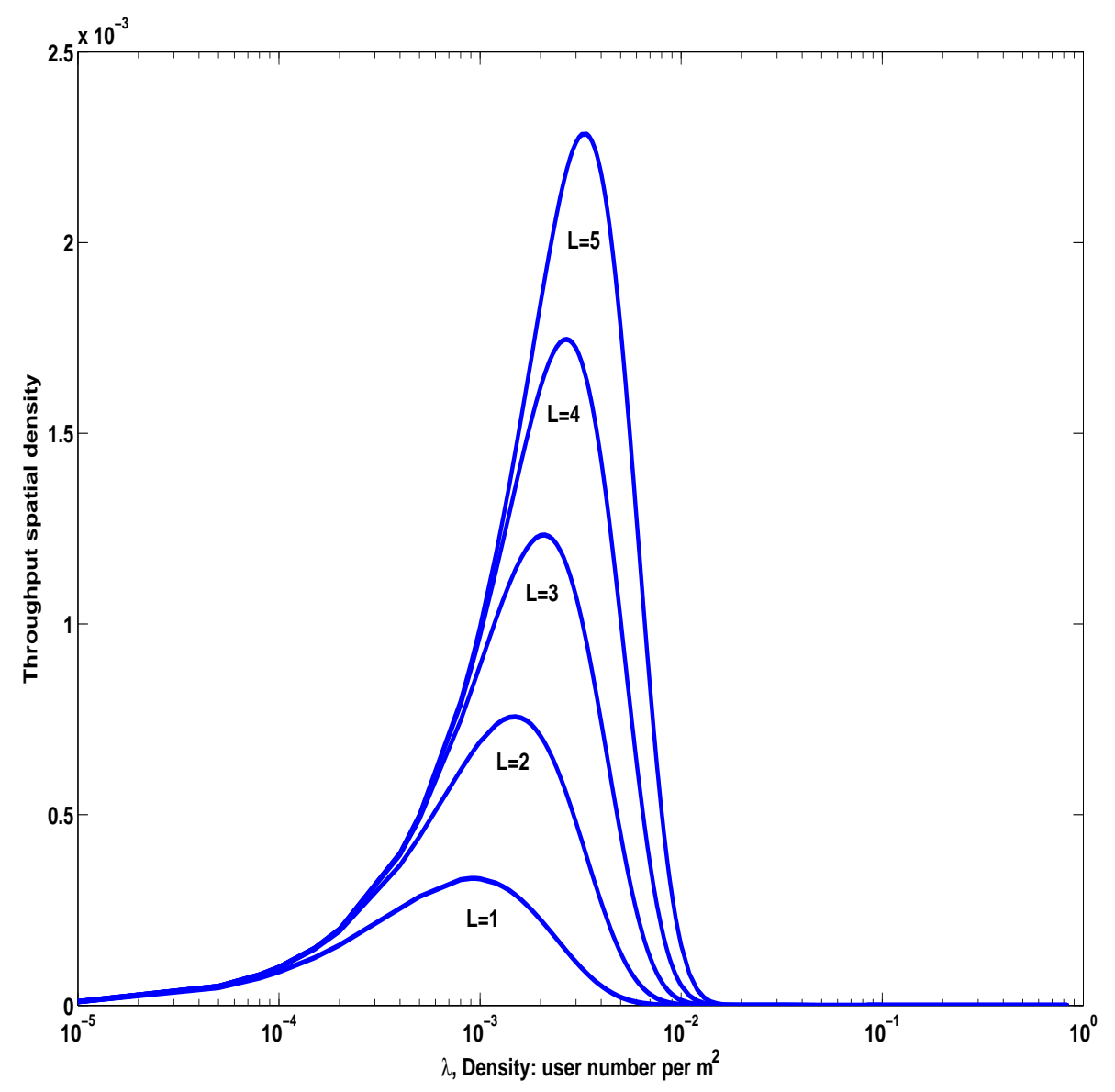

Fig. 3: Optimum combining: Throughput spatial density with $L=1 \cdots 5, \sigma^{2}=$ $-57 d B /$ antenna. 


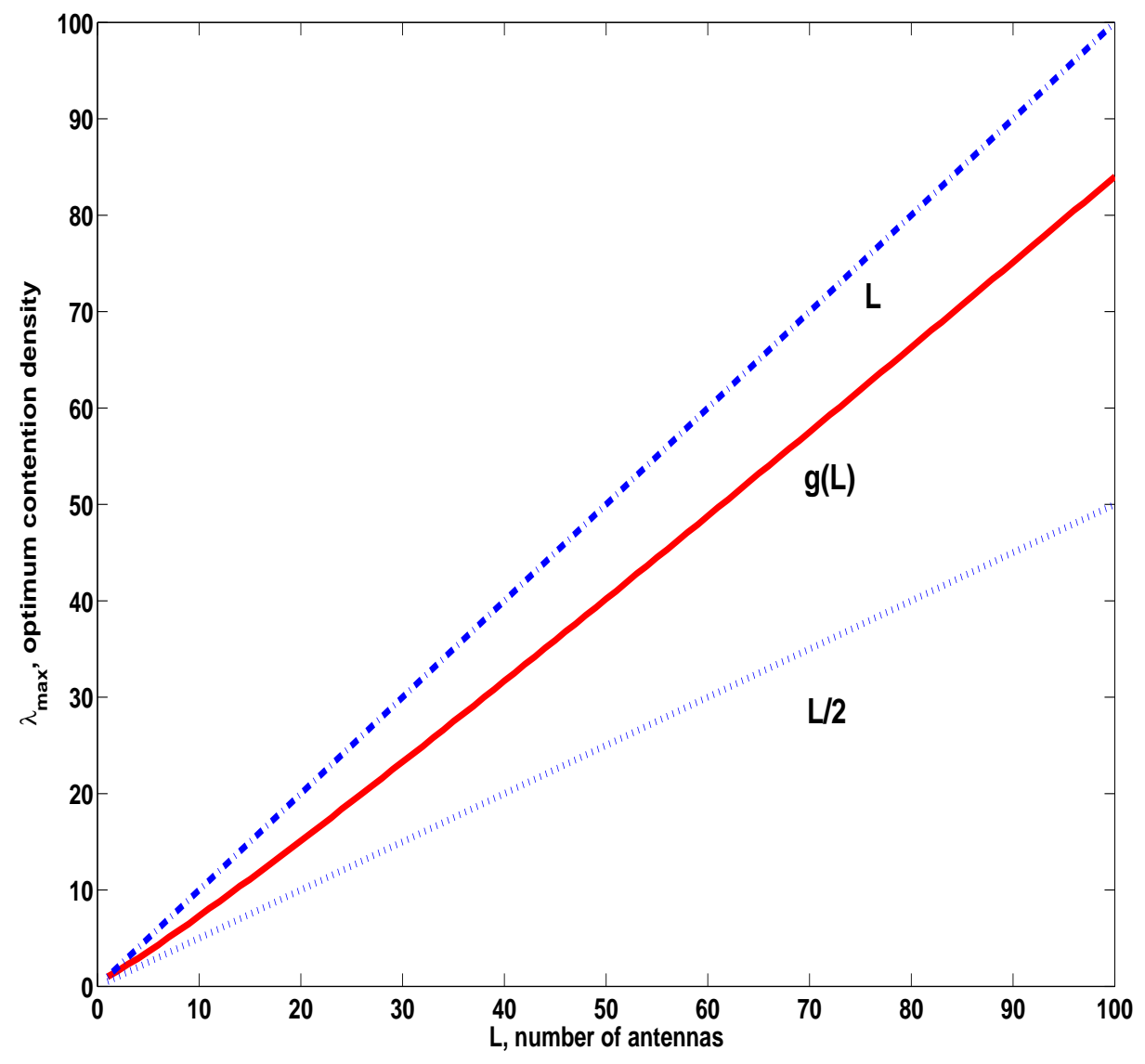

Fig. 4: Optimum combining: Optimum contention density as a function of the antenna array dimension, with $\sigma^{2}=0$ and $\Delta \gamma^{2 / \alpha}$ normalized to 1 . 